\title{
RESEARCH
}

Open Access

\section{The interplay between host genetics and the gut microbiome reveals common and distinct microbiome features for complex human diseases}

Fengzhe $\mathrm{Xu}^{1 \dagger}$, Yuanqing Fu ${ }^{1,3+}$, Ting-yu Sun ${ }^{2}$, Zengliang Jiang ${ }^{1,3}$, Zelei Miao ${ }^{1}$, Menglei Shuai ${ }^{1}$, Wanglong Gou ${ }^{1}$, Chu-wen Ling ${ }^{2}$, Jian Yang ${ }^{4,5}$, Jun Wang ${ }^{6^{*}}$, Yu-ming Chen ${ }^{2^{*}}$ and Ju-Sheng Zheng ${ }^{1,3,7^{*}}$ (i)

\begin{abstract}
Background: Interest in the interplay between host genetics and the gut microbiome in complex human diseases is increasing, with prior evidence mainly being derived from animal models. In addition, the shared and distinct microbiome features among complex human diseases remain largely unclear.

Results: This analysis was based on a Chinese population with 1475 participants. We estimated the SNP-based heritability, which suggested that Desulfovibrionaceae and Odoribacter had significant heritability estimates ( 0.456 and 0.476 , respectively). We performed a microbiome genome-wide association study to identify host genetic variants associated with the gut microbiome. We then conducted bidirectional Mendelian randomization analyses to examine the potential causal associations between the gut microbiome and complex human diseases. We found that Saccharibacteria could potentially decrease the concentration of serum creatinine and increase the estimated glomerular filtration rate. On the other hand, atrial fibrillation, chronic kidney disease and prostate cancer, as predicted by host genetics, had potential causal effects on the abundance of some specific gut microbiota. For example, atrial fibrillation increased the abundance of Burkholderiales and Alcaligenaceae and decreased the abundance of Lachnobacterium, Bacteroides coprophilus, Barnesiellaceae, an undefined genus in the family Veillonellaceae and Mitsuokella. Further disease-microbiome feature analysis suggested that systemic lupus erythematosus and chronic myeloid leukaemia shared common gut microbiome features.
\end{abstract}

Conclusions: These results suggest that different complex human diseases share common and distinct gut microbiome features, which may help reshape our understanding of disease aetiology in humans.

Keywords: Gut microbiome, Host genetics, Bidirectional Mendelian randomization analyses, Disease-microbiome features

\footnotetext{
* Correspondence: junwang@im.ac.cn; chenyum@mail.sysu.edu.cn; zhengjusheng@westlake.edu.cn

${ }^{\dagger}$ Fengzhe $\mathrm{Xu}$ and Yuanqing Fu contributed equally to the work.

${ }^{6}$ CAS Key Laboratory for Pathogenic Microbiology and Immunology, Institute

of Microbiology, Chinese Academy of Sciences, Beijing, China

${ }^{2}$ Guangdong Provincial Key Laboratory of Food, Nutrition and Health,

Department of Epidemiology, School of Public Health, Sun Yat-sen University,

Guangzhou, China

'Zhejiang Provincial Laboratory of Life Sciences and Biomedicine, Key

Laboratory of Growth Regulation and Translational Research of Zhejiang

Province, School of Life Sciences, Westlake University, Hangzhou, China

Full list of author information is available at the end of the article
}

C C The Author(s). 2020 Open Access This article is licensed under a Creative Commons Attribution 4.0 International License, which permits use, sharing, adaptation, distribution and reproduction in any medium or format, as long as you give appropriate credit to the original author(s) and the source, provide a link to the Creative Commons licence, and indicate if changes were made. The images or other third party material in this article are included in the article's Creative Commons licence, unless indicated otherwise in a credit line to the material. If material is not included in the article's Creative Commons licence and your intended use is not permitted by statutory regulation or exceeds the permitted use, you will need to obtain permission directly from the copyright holder. To view a copy of this licence, visit http://creativecommons.org/licenses/by/4.0/. The Creative Commons Public Domain Dedication waiver (http://creativecommons.org/publicdomain/zero/1.0/) applies to the data made available in this article, unless otherwise stated in a credit line to the data. 


\section{Background}

Ever increasing evidence has suggested that the gut microbiome is involved in many physiological processes, such as energy harvesting, the immune response and neurological function [1-3]. With successes of investigation into the clinical application of faecal transplants, the modulation of the gut microbiome has emerged as a potential treatment option for some complex diseases, including inflammatory bowel disease and colorectal cancer $[4,5]$. However, it is still unclear whether the gut microbiome has the potential to be clinically applied for the prevention or treatment of many other complex diseases. Therefore, it is important to clarify the bidirectional causal association between the gut microbiome and complex human diseases or traits.

Mendelian randomization (MR) is a method that uses genetic variants as instrumental variables to investigate the causality between an exposure and an outcome in observational studies [6]. Prior studies provide evidence that the composition or structure of the gut microbiome can be influenced by host genetics [7-10]. On the other hand, host genetic variants associated with the gut microbiome are rarely explored in Asian populations; thus, we still lack instrumental variables to perform MR for the gut microbiome in Asians. This calls for a novel microbiome genome-wide association study (GWAS) in Asian populations.

Along with the causality issue between the gut microbiome and complex human diseases, it is unclear whether complex human diseases have similar or unique gut microbiome features. The identification of common and distinct gut microbiome features across different diseases may shed light on novel relationships among the complex diseases and update our understanding of the disease aetiology in humans. However, the composition and structure of the gut microbiome are influenced by a variety of factors, including the environment, diet and regional variation [11-13], which poses a key challenge for the description of representative microbiome features for a specific disease. Although there were several studies comparing disease-related gut microbiome features [14-16], few of them examined and compared the microbiome features across different complex human diseases.

In the present study, we performed a microbiome GWAS in a Chinese cohort, the Guangzhou Nutrition and Health Study (GNHS) [17], including 1475 participants. Subsequently, we applied a bidirectional MR method to explore the genetically predicted relationship between the gut microbiome and complex human diseases. To explore novel relationships among complex human diseases based on the gut microbiome, we investigated the shared and distinct gut microbiome features across diverse complex human diseases.

\section{Results}

Overview of the study

Our study was based on the GNHS, with 4048 participants (40-75 years old) living in the urban area of Guangzhou city recruited during 2008 and 2013 [17]. In the GNHS, stool samples were collected among 1937 participants during follow-up visits, among whom 1475 unrelated participants not taking antibiotics were included in our discovery microbiome GWAS. We then included an additional 199 participants with both genetic data and gut microbiome data as a replication cohort, which belonged to the control arm of a case-control study of hip fracture in Guangdong Province, China [18] (see also Fig. 1).

\section{SNP-based heritability of the gut microbiome}

The heritability of alpha diversity ranged from 0.035 to 0.103 (SE: from 0.174 to 0.193 , Supplementary Table S3). Significant heritability estimates were observed for several taxa (see also Fig. 2, Supplementary Table S3), with crude $p$ values $<0.05$. To further correct the multiple testing, we calculated the effective number of independent taxa in each taxonomic level (phylum level: 2.3, class level: 2.9 , order level: 2.9 , family level: 5.5 , genus level: 5.6, species level: 3.2 ), as some taxa were highly correlated with each other. The results suggested that Desulfovibrionaceae and Odoribacter were heritable $(p<$ $0.05 / n$, where $n$ is the effective number of independent taxa). Notably, among the suggestively heritable taxa in our cohort [Paraprevotellaceae], Veillonellaceae, Desulfovibrionaceae, Pasteurellaceae, Odoribacter, Paraprevotella, Veillonella and Bifidobacterium had nominally significant heritability estimates in prior literature $[7$, 31-33].

\section{Association of host genetics with gut microbiome features}

We generated categorical variable enterotypes (Prevotella vs. Bacteroides) of the participants based on the genus-level relative abundance of the gut microbiome [34]. Thereafter, we performed a GWAS for enterotypes using a logistic regression model to explore potential associations between host genetics and enterotypes. However, we did not find any genome-wide significant loci $(p$ $\left.<5 \times 10^{-8}\right)$.

To examine the association of host genetic variants with alpha diversity, we performed a GWAS for four indices (Shannon diversity index, Chaol diversity indices, observed OTU index and phylogenetic diversity), but again, no genome-wide significant signal $\left(p<5 \times 10^{-8}\right)$ was found. To further investigate whether there is a host genetic basis underlying alpha diversity, we constructed a polygenic score for each alpha diversity indicator in the replication cohort using the genetic variants that 


\section{A. Association of host genetics with gut microbiome in a Chinese population.}
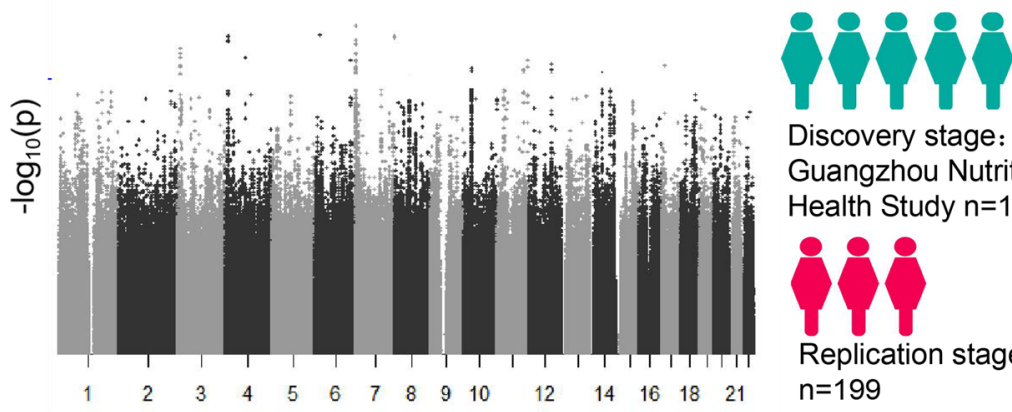

Discovery stage:

Guangzhou Nutrition and

Health Study $n=1,475$

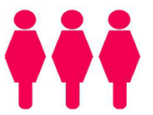

Replication stage:

$\mathrm{n}=199$

\section{B. The causal relationships between gut microbiome and complex human diseases.}

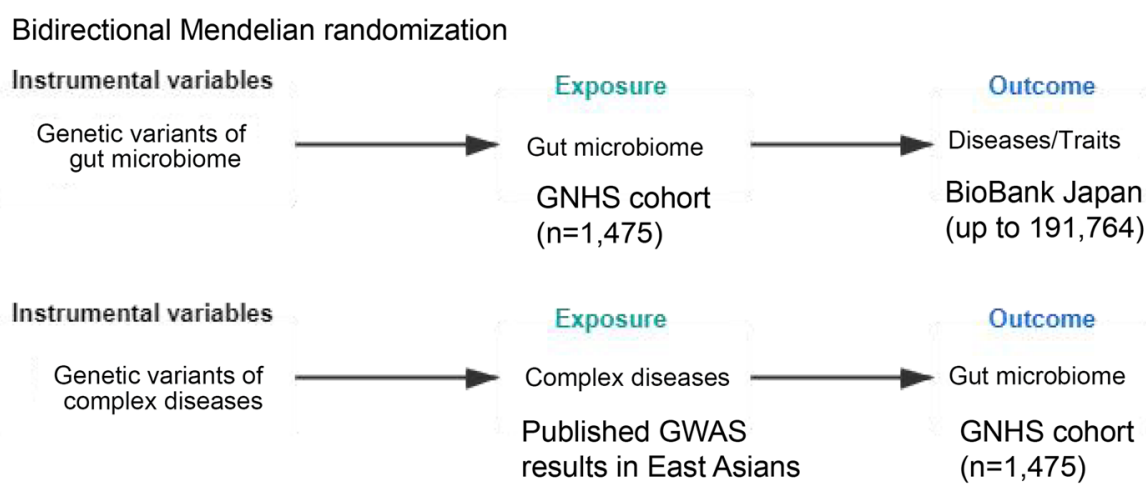

\section{The shared and distinct microbiome features among complex human diseases.}

1,919 participants from GNHS cohort.

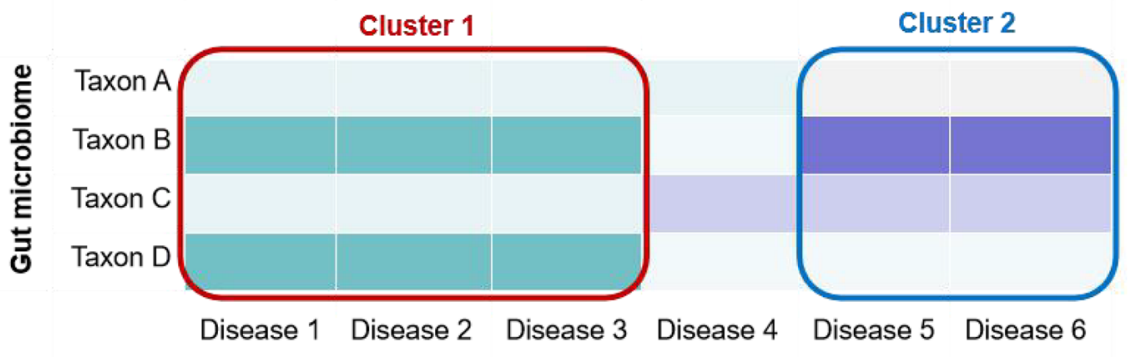

Fig. 1 Study overview. The figure shows the highlights of our study. First, we performed a microbiome genome-wide association study in a Chinese population (step A). We validated significant genetic variants reported in previous studies and replicated our results in an independent cohort. Second, we investigated the causal relationship between the gut microbiome and complex human diseases using host genetics as instrumental variables for bidirectional Mendelian randomization (MR) analysis (step B). For the analysis of the effects of the gut microbiome on complex traits, we used publicly available GWAS summary statistics of complex traits $(n=58)$ and diseases (type 2 diabetes mellitus (T2DM), atrial fibrillation (AF), colorectal cancer (CRC) and rheumatoid arthritis) reported by BioBank Japan [19-24]. For the reverse MR analyses, the diseases of interest included T2DM (cases: 7,109; non-cases: 86,022), AF (cases: 8,180; non-cases: 28,612), coronary artery disease (cases: 1,515; non-cases: 5019), chronic kidney disease $(n=71,149)$, Alzheimer's disease (cases: 477; non-cases: 442), CRC (cases: 8027; non-cases: 22,577) and prostatic cancer (cases: 495; non-cases: 640) reported in the previous large-scale GWASs in East Asians [19, 25-30]. Finally, we identified common and distinct gut microbiome features across different diseases (step C) 


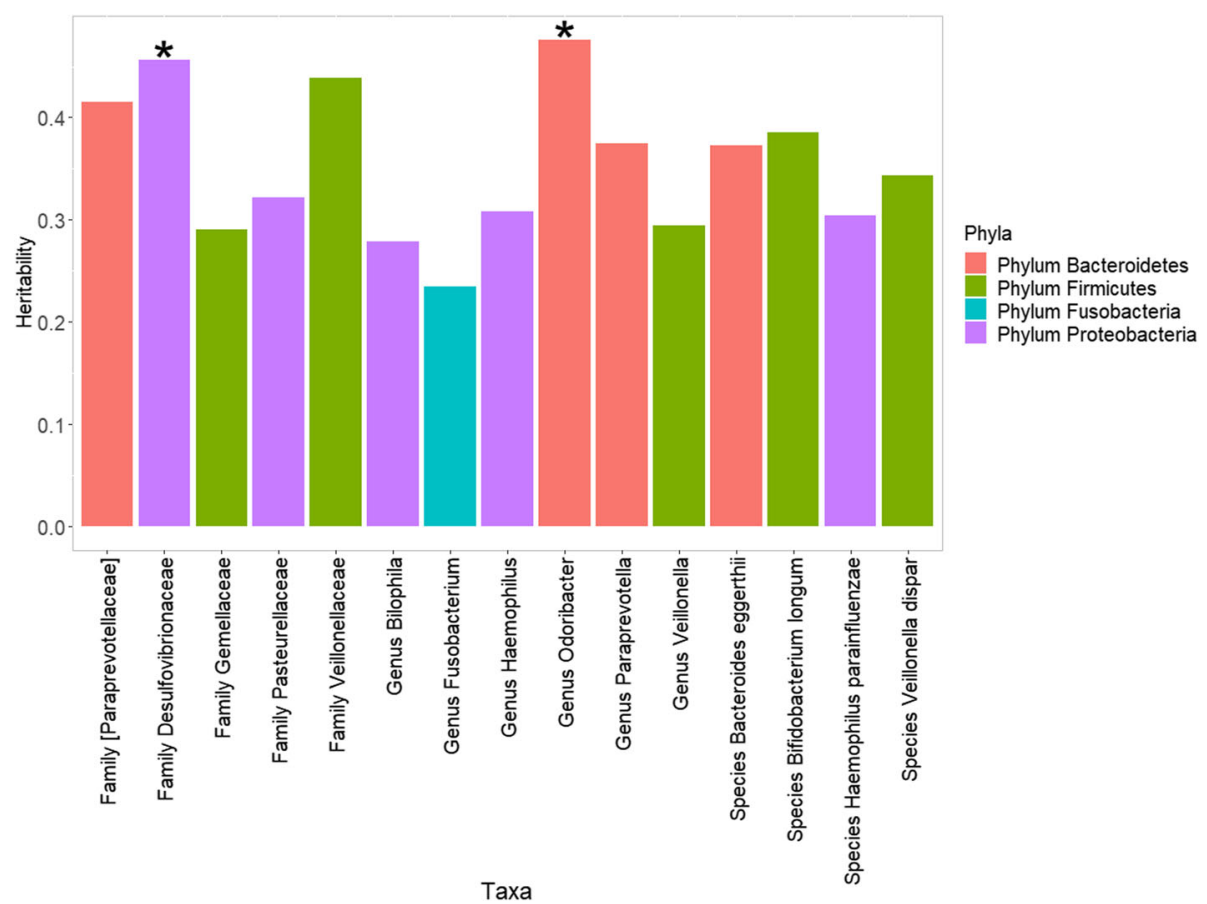

Fig. 2 The SNP-based heritability of the gut microbiome. The plot shows the taxa with nominally significant heritability estimates $(p<0.05)$. ${ }^{*} p<0.05 / n$, where $n$ is the effective number of independent taxa in each taxonomic level

showed suggestive significance $\left(p<5 \times 10^{-5}\right)$ in the discovery GWAS. The polygenic score was not significantly associated with its corresponding alpha diversity index in our replication cohort. Furthermore, none of the associations with alpha diversity indices reported in the literature could be replicated (Supplementary Table S8) [7].

The beta diversity GWAS was performed with MicrobiomeGWAS based on Bray-Curtis dissimilarity [35]. We found that one locus at the SMARCA2 gene (rs6475456) was associated with beta diversity at a genome-wide significant level $\left(p=3.96 \times 10^{-9}\right)$. However, we could not replicate the results in the replication cohort, which might be due to the limited sample size of the replication cohort. In addition, prior literature had reported 73 genetic variants that were associated with beta diversity [8, 13, 36, 37], among which we found that 3 single-nucleotide polymorphisms (SNPs, UHRF2 geners563779, LHFPL3 gene-rs12705241, CTD-2135J3.4rs11986935) had nominally significant $(p<0.05)$ associations with beta diversity in our cohort (Supplementary Table S7), although none of the associations survived Bonferroni correction. These studies used various methods for the sequencing and calculation of beta diversity, which raised challenges to verify and extrapolate results across populations.

We subsequently performed a discovery GWAS for individual gut microbes in our own GNHS discovery dataset. For the taxa $(n=114)$ present in not fewer than $90 \%$ of participants, we carried out an analysis based on a log-normal model. For other taxa $(n=88)$ present in fewer than $90 \%$, we transformed the absence/presence of the taxon into binary variables and used a logistic model to prevent zero inflation (Supplementary Table S1). For all the gut microbiome taxa, the significance threshold was defined as $5 \times 10^{-8}$ in the discovery stage. We found that 6 taxa were associated with host genetic variants in the discovery cohort $\left(p<5 \times 10^{-8} / n\right.$, where $n$ is the effective number of independent taxa in each taxonomic level, Supplementary Table S5); however, these associations were not significant $(p>0.05)$ in the replication cohort. We then took the genetic loci reported to be associated with individual taxa in prior studies $[7,8,13$, 37] for replication in our GNHS dataset. Although none of the associations of these genetic variants with taxa survived the Bonferroni correction $\left(p<1 \times 10^{-4}\right)$, we found that STPG2-rs4699323 had a nominally significant association $(p<0.05)$ with Clostridiales (Beta: -0.131 [95\% CI $-0.233,-0.029], p=0.012$; Supplementary Table S6). We then used a threshold of $p<5 \times 10^{-5}$ at the discovery GWAS stage to incorporate additional genetic variants that might explain a larger proportion of heritability for taxa, and based on this, we constructed a polygenic score for each taxon in the replication. We found that the polygenic scores were significantly associated with 5 taxa, including Saccharibacteria (also known 
as TM7 phylum), Clostridiaceae, Comamonadaceae, Klebsiella and Desulfovibrio d168, in the replication set $(p<0.05$, Methods, see also Supplementary Figure S1, Supplementary Table S9).

\section{Genetic correlation of gut microbiome and traits}

While the associations of the microbiome with complex diseases and traits have been widely reported [38], the genetic correlation between the gut microbiome and traits of interest is less clear. Therefore, we applied bivariate GREML analysis to address this question. The traits included BMI, fasting blood sugar (FBS), glycosylated haemoglobin (HbA1c), systolic blood pressure (SBP), diastolic blood pressure (DBP), high-density lipoprotein cholesterol (HDL-C), low-density lipoprotein cholesterol (LDL-C), total cholesterol (TC) and triglyceride (TG), none of which could pass Bonferroni correction. HDL-C was the only trait that had nominal genetic correlation $(p<0.05)$ with gut microbes (specifically, Desulfovibrionaceae and [Prevotella], Supplementary Table S4).

\section{Bidirectional assessment of the genetically predicted association between the gut microbiome and complex diseases/traits}

Using genetic-variant-composed polygenic scores as genetic instruments, we performed MR analysis to assess the putative causal effect of the microbiome (Saccharibacteria, Clostridiaceae, Comamonadaceae, Klebsiella and Desulfovibrio d168) on complex human diseases or traits. The inverse variance weighted (IVW) method was used for the MR analysis, while the other three methods (weighted median, MR-Egger and MR-PRESSO) were applied to confirm the robustness of the results. Horizontal pleiotropy was assessed using the MR-PRESSO global test and MR-Egger regression. For the analysis of the gut microbiome on complex traits, we downloaded publicly available GWAS summary statistics of complex traits $(n=58)$ and diseases (type 2 diabetes mellitus (T2DM), atrial fibrillation (AF), colorectal cancer (CRC) and rheumatoid arthritis (RA)) reported by BioBank Japan [19-24]. The results suggested that Saccharibacteria (per 1-SD higher in the log-transformed abundance) could potentially decrease the concentration of serum creatinine $(-0.011$ [95\% CI $-0.019,-0.003], p=$ $0.007)$ and increase the estimated glomerular filtration rate (eGFR) (0.012 [95\% CI 0.004, 0.020], $p=0.003$, Supplementary Table S10), which might help improve renal function. We did not find evidence of pleiotropic effects: genetic variants associated with Saccharibacteria were not associated with any of the above traits (58 complex traits and 4 disease outcomes, $p<0.05 / 62)$. These taxa were not causally associated with other complex diseases or traits in our MR analyses, which might be due to the limited genetic instruments discovered in our present study.

We subsequently performed a reverse MR analysis to assess the potential causal effect of complex human diseases on gut microbiome features. For the reverse MR analyses, the diseases of interest included T2DM, AF, coronary artery disease (CAD), chronic kidney disease (CKD), Alzheimer's disease (AD), CRC and prostatic cancer $(\mathrm{PCa})$, and their instrumental variables for the MR analysis were based on previous large-scale GWASs in East Asians [19, 25-30]. The results suggested that AF and CKD were causally associated with the gut microbiome (see also Fig. 3a, b, Supplementary Table S11). Specifically, genetically predicted higher risk of AF (per log odds) was associated with a lower abundance of Lachnobacterium (Beta: - 0.078 [95\% CI - 0.148, 0.006], $p=0.034$ ), Bacteroides coprophilus (Beta: -0.113 [95\% CI - 0.184, - 0.041], $p=0.002)$, Barnesiellaceae (odds ratio: 0.818 [95\% CI 0.686, 0.976], $p=0.026$ ), an undefined genus in the family Veillonellaceae (odds ratio: 0.801 [95\% CI 0.669, 0.960], $p=0.017$ ) and Mitsuokella (odds ratio: 0.657 [95\% CI 0.496, 0.870], $p=0.003$ ), and higher abundance of Burkholderiales (Beta: 0.079 [95\% CI 0.009, 0.150], $p=0.027$ ) and Alcaligenaceae (Beta: 0.082 [95\% CI 0.012, 0.152], $p=0.022$ ). Additionally, genetically predicted higher risk of CKD could increase Anaerostipes (Beta: 0.291 [95\% CI 0.057, 0.524], $p$ $=0.015$ ) abundance, and a higher risk of PCa could decrease [Prevotella] (odds ratio: - 0.758 [95\% CI - 1.354, -0.162], $p=0.013)$.

\section{Microbiome features of complex human diseases}

To further investigate the potential complex diseases that may be correlated with the taxa affected by AF, we applied Phylogenetic Investigation of Communities by Reconstruction of Unobserved States (PICRUSt) to predict the disease pathway abundance [39]. We used Spearman's rank-order correlation to test whether the relative abundances of predicted diseases based on PICRUSt were associated with the aforementioned AF-associated taxa (see also Supplementary Figure S2, Supplementary Table S12). The heatmap indicated that cancers and neurodegenerative diseases, including Parkinson's disease (PD), AD, amyotrophic lateral sclerosis (ALS) and AF, were correlated with similar gut microbiomes. Although the association among these diseases is highly supported by previous studies [40-42], no study has compared common gut microbiome features across these different diseases.

To compare gut microbiome features across human diseases, we used the predicted disease abundance based on PICRUSt and performed k-medoid clustering. According to the optimum average silhouette width [43], we chose the optimal number of clusters for further analysis. The plot showed that neurological diseases, 


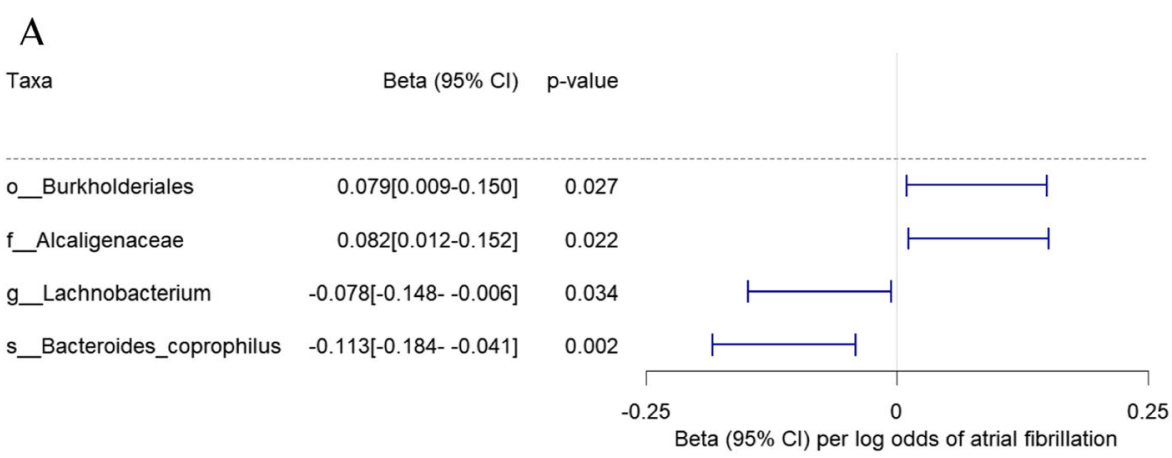

B

Taxa $\quad$ OR $(95 \% \mathrm{Cl}) \quad \mathrm{p}$-value

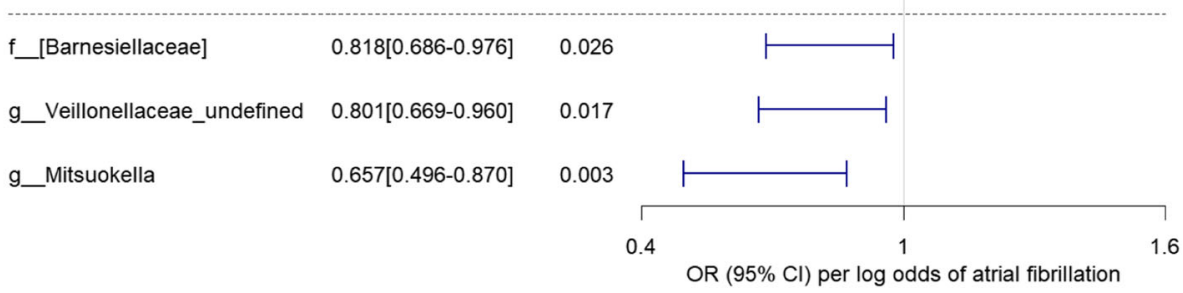

Fig. 3 Effect of host genetically predicted higher atrial fibrillation risk on the gut microbiome. a Causal association of atrial fibrillation with the abundance of Burkholderiales, Alcaligenaceae, Lachnobacterium and Bacteroides coprophilus. The magnitude of the effect of atrial fibrillation on taxa is dependent on changes in the abundance of bacteria (1-SD of the log-transformed abundance) per genetically determined higher log odds of atrial fibrillation. $\mathbf{b}$ Causal association of atrial fibrillation with the presence of Barnesiellaceae, an undefined genus in the family Veillonellaceae and Mitsuokella. The magnitude of the effect of atrial fibrillation on taxa is presented as an odds ratio increase in the log odds of atrial fibrillation

including ALS and AD, belonged to the same cluster, while PD and CRC had much similarity in the gut microbiome. The results also suggested that systemic lupus erythematosus (SLE) and chronic myeloid leukaemia (CML) shared similar gut microbiome features (see also Fig. 4a, b). Moreover, we could replicate these clusters in our replication cohort, which suggested that the clustering results were robust (see also Fig. 4c).

We further asked whether the gut microbiome contributed to the novel clustering. To this end, we repeated the analysis among participants who took antibiotics less than two weeks before stool sample collection, considering that antibiotic treatments were believed to cause microbiome imbalance. We used the Jaccard similarity coefficient to estimate the cluster difference among the GNHS cohort, the replication cohort and the antibiotic group. The similarity between the GNHS cohort and the replication cohort was higher than that between the GNHS cohort and the antibiotic group (Jaccard similarity coefficient: 0.61 vs. 0.11 ). The results indicated a different clustering, which suggested that the gut microbiome indeed contributed to the correlations among diseases (see also Fig. 4d). To further demonstrate common microbiome features across different diseases, we examined the correlation of the predicted diseases with genus-level taxa. The results showed that complex human diseases shared similar gut microbiome features, as well as distinct features on their own (see also Fig. 5, Supplementary Table S13).

To validate whether the disease-related gut microbiome features annotated by KEGG would be associated with the risk of the disease in a real-world communitybased cohort, we used T2DM as an example, examining the association of predicted T2DM-related microbiome features with T2DM risk in our GNHS cohort. We constructed a microbiome risk score (MRS) based on 16 selected taxa with predicted correlation coefficients with T2DM greater than 0.2. A logistic regression model was used to examine the association between MRS and T2DM risk in GNHS ( $n=1886$, with 217 T2DM cases). The results showed that MRS was positively associated with the risk of T2DM (odds ratio: 1.176 [95\% CI 1.114, 1.244], $\left.p=8.75 \times 10^{-9}\right)$.

\section{Discussion}

Our study is among the first to investigate host geneticsgut microbiome associations in East Asian populations and reveals that several microbiome species (e.g., Saccharibacteria and Klebsiella) are influenced by host genetics. We found that Saccharibacteria might causally 

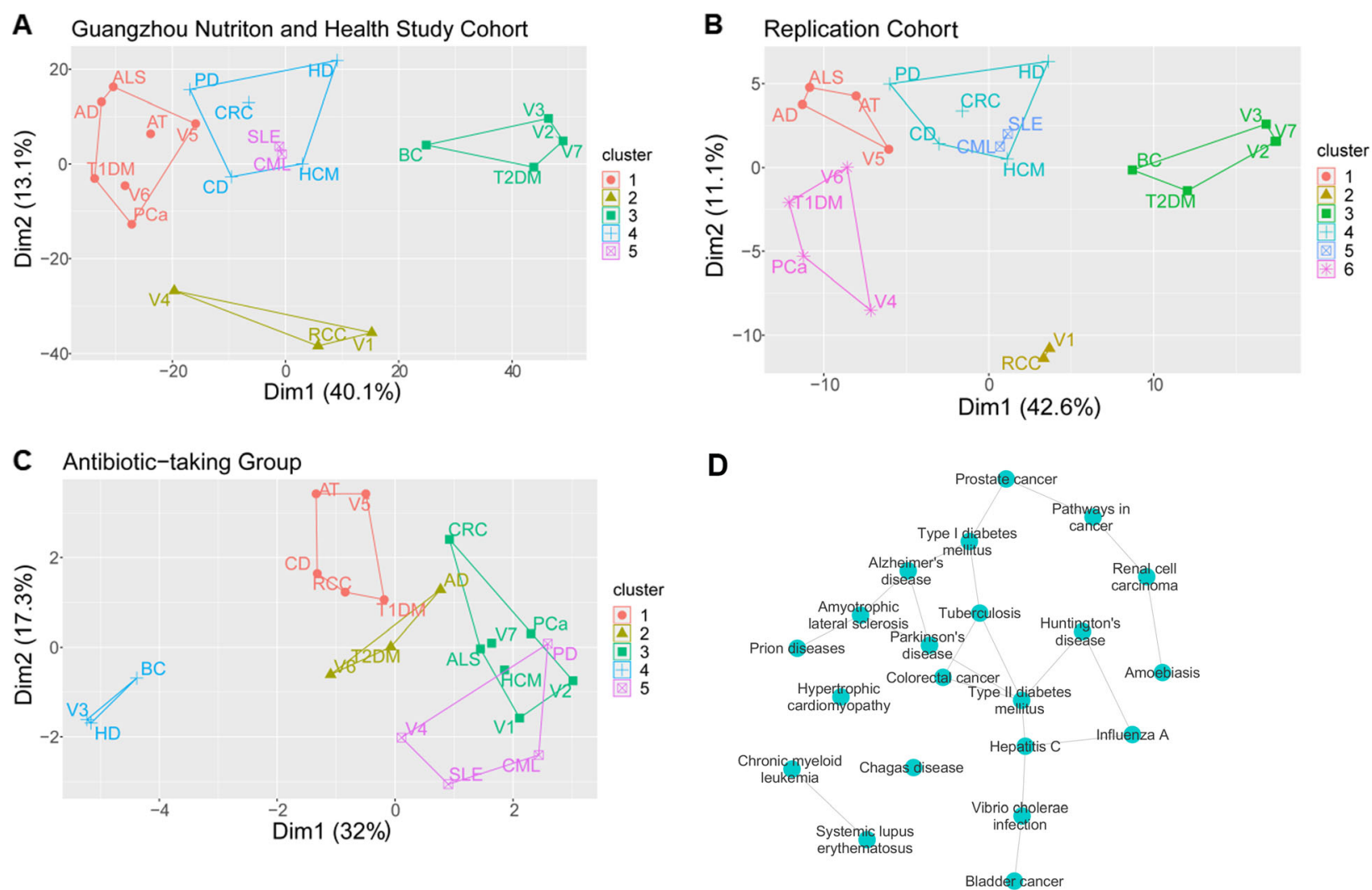

Fig. 4 Association and cluster of diseases predicted by the gut microbiome. a Plot of clusters in the Guangzhou Nutrition and Health Study (GNHS) cohort $(n=1919)$. b Plot of cluster results in the replication cohort $(n=217)$. $\mathbf{c}$ Plot of 5 clusters in antibiotic-taking participants $(n=18)$. The optimal cluster was 5 in the GNHS cohort and 6 in the replication cohort. The clusters share consistent components between the two studies. In contrast, components are different between antibiotic-taking participants and control groups. Dimension1 (Dim1) and dimension2 (Dim2) explained $40.1 \%$ and $13.1 \%$ of the variance, respectively, in the GNHS cohort. The annotation for variables is as follows. AT African trypanosomiasis, AD Alzheimer's disease, V1 amoebiasis, ALS amyotrophic lateral sclerosis, BC bladder cancer, CD Chagas disease, CML chronic myeloid leukaemia, CRC colorectal cancer, V2 hepatitis C, HD Huntington's disease, HCM hypertrophic cardiomyopathy, V3 influenza A, PD Parkinson's disease, V4 pathways in cancer, V5 Prion disease, PCa prostate cancer, RCC renal cell carcinoma, SLE systemic lupus erythematosus, V6 tuberculosis, T1DM type I diabetes mellitus, T2DM type II diabetes mellitus, V7 Vibrio cholerae infection. $\mathbf{d}$. Gut microbiome-predicted network of relationships among different complex human diseases. The relationship between diseases is determined by SPIEC-EASI with non-normalized predicted abundance data. The diseases that shared the same edge had the gut microbiome-predicted correlation

improve renal function by affecting renal function biomarkers (i.e., creatinine and eGFR). On the other hand, complex diseases such as atrial fibrillation, chronic kidney disease and prostate cancer have potential causal effects on the gut microbiome. More interestingly, our results indicated that different complex diseases may be mechanistically correlated by sharing common gut microbiome features but also maintaining their own distinct microbiome features.

Previous studies and our study showed that the gut microbiome had an inclination to be influenced by host genetics $[8,10,37,44,45]$. The results suggested that Desulfovibrionaceae and Odoribacter had nominally significant heritability estimates, which were consistent with prior results [7]. We also identified several suggestively heritable taxa that were nominally significant in previous studies [31-33]. In addition, we successfully constructed polygenic scores for Clostridiaceae and Comamonadaceae, both of which have been identified to be heritable or suggested to be heritable $[7,45]$.

We could not replicate any of the reported genetic variants that were significantly associated with gut microbiome features in prior reports, which might be due to multiple reasons. One of the major reasons may be that the massive multiple testing in insufficiently large samples in prior microbiome GWASs may potentially lead to false-positive findings. In addition, other factors, including ethnic differences, heterogeneity between studies, gene-environment interactions and dissimilarity in sequencing methods, might also make it difficult to extrapolate results from microbiome GWASs across populations in the microbiome field. Nevertheless, we successfully replicated several polygenic scores of the gut microbiome. The current study represents the 


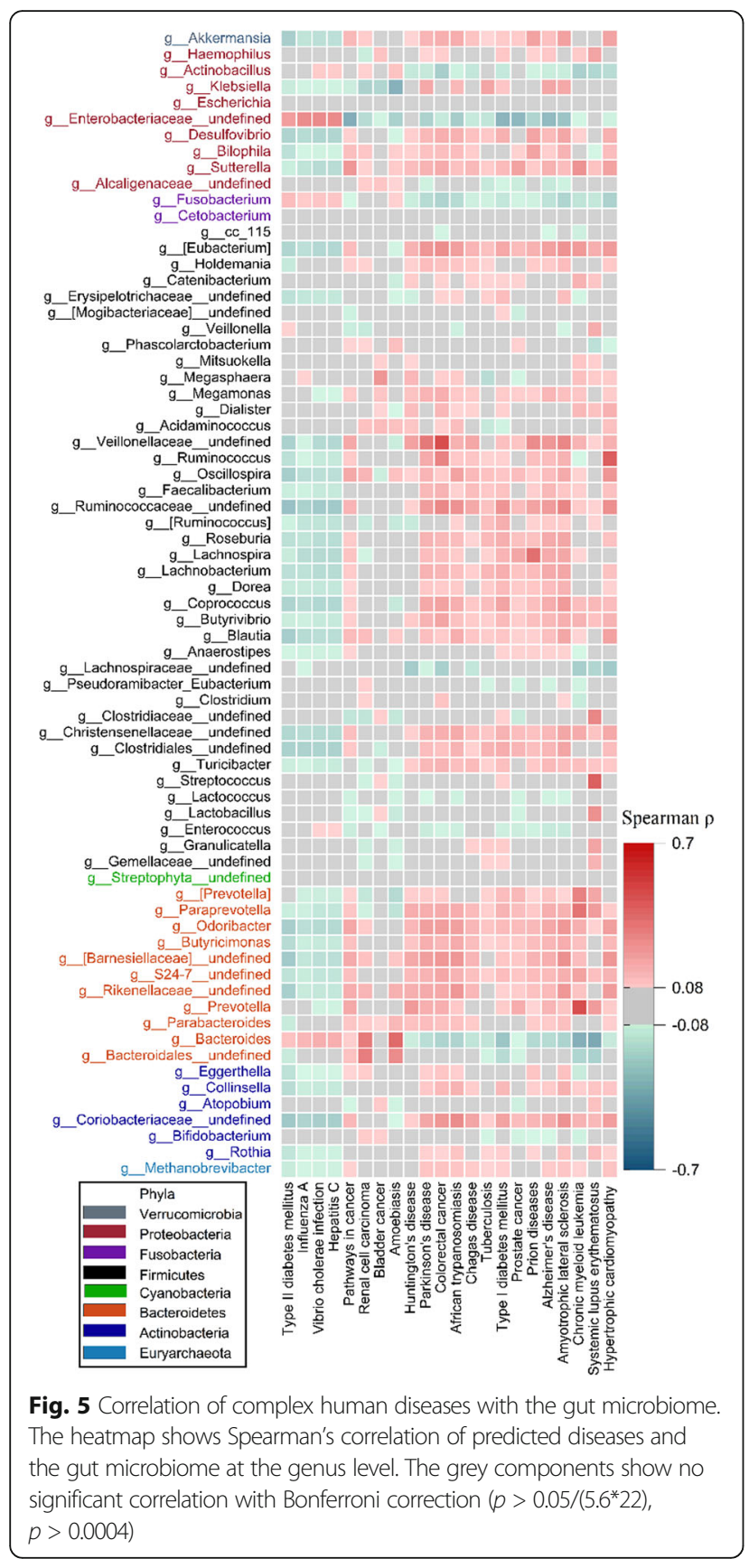

largest dataset, to the best of our knowledge, in Asian populations and may serve as a unique resource for large-scale trans-ethnic meta-analyses of microbiome GWASs in the future.

MR analysis showed that Saccharibacteria might decrease the concentration of serum creatinine and increase eGFR. Little is known about Saccharibacteria as one of the uncultivated phyla, and previous studies have shown that it might be essential for the immune response, oral inflammation and inflammatory bowel disease [46-48]. Our results also provided a genetic instrument of Saccharibacteria for further causal analysis with other complex diseases. The reverse MR analysis provided evidence that $\mathrm{AF}, \mathrm{CKD}$ and $\mathrm{PCa}$ could causally influence the gut microbiome. The rare and low-frequency variants may have an important impact on common diseases [49]; thus, it will be of interest to clarify the effects of low-frequency variants on the gut microbiome in cohorts with large sample sizes in the future.

Our results indicate that the gut microbiome helps reveal novel and interesting relationships among complex human diseases, and different diseases may have common and distinct gut microbiome features. A prior study including participants from different countries identified three microbiome clusters [34]. Notably, this study focused on classifying the individuals into distinct enterotypes regardless of the individuals' health status, while in the present study, we described representative microbiome features for diseases of interest. We provided an approach to interpret the data from mechanistic studies based on the microbiome. The microbiome features revealed a close association of AF with neurodegenerative diseases as well as cancers, which was supported by prior studies showing that $\mathrm{AF}$ had a correlation with $\mathrm{AD}$ and PD [40, 41], and AF patients had relatively higher risks of several cancers, including lung cancer and CRC [42, 50]. We also observed that the microbiome features of SLE and CML were highly similar. Interestingly, a tyrosine kinase inhibitor of platelet-derived growth factor receptor, imatinib, was widely used to treat CML and significantly ameliorated survival in murine models of SLE [51]. In addition, a close association between CRC and PD has also been reported in several observational cohorts [52, 53]. Collectively, these findings strongly supported our hypothesis that complex human diseases sharing similar microbiome features might be mechanistically correlated. Furthermore, from the perspectives of risk genes of $\mathrm{AF}$ and neurodegenerative diseases, previous GWASs for AF identified two loci at PITX2 geners6843082 and C9orf3 gene-rs7026071, which were also associated with a risk of ALS ( $p=0.0138$ and $p=0.049$, respectively) [54-56].

We acknowledge several limitations of our study. First, the participants were of East Asian ancestry; thus, factors such as ethnic differences and gene-environment interactions might make it difficult to generalize the prior results to our study and extrapolate our results to different ethnic populations. Second, although our analysis included participants with the identical by descent (IBD) $<0.185$, the vertical transmission of the microbiome from parent to offspring might still partially affect the SNP-based heritability estimates and polygenic scores $[32,57]$. Third, genetic factors could explain only a small proportion of the variance in gut microbiome features; 
thus, the power to detect the causal relationship was limited. Therefore, large-scale studies are warranted to reveal potential relationships between the gut microbiome and complex traits.

\section{Conclusions}

In summary, we reveal some causal relationships between the gut microbiome and complex human diseases or traits. The disease and gut microbiome feature analysis revealed novel relationships among complex human diseases, which may help reshape our understanding of disease aetiology and provide some clues for extending the clinical indications of existing drugs for different diseases.

\section{Method}

\section{Study participants and sample collection}

Our study was based on the Guangzhou Nutrition and Health Study (GNHS), with 4048 participants (40-75 years old) living in the urban area of Guangzhou city recruited during 2008 and 2013 [17]. We followed up with participants every 3 years. In the GNHS, stool samples were collected from 1937 participants during follow-up visits. Among those with stool samples, 1717 participants had genetic data, and IBD for 1475 participants was less than 0.185

We included 199 participants with both genetic data and gut microbiome data as a replication cohort, which belonged to the control arm of a case-control study of hip fracture with the participants (52-83 years old) recruited between June 2009 and August 2015 in Guangdong Province, China [18].

Blood samples of all participants were collected after overnight fasting, and the buffy coat was separated from whole blood and stored at $-80^{\circ} \mathrm{C}$. Stool samples were collected during the on-site visit of the participants at Sun Yat-sen University. All samples were manually stirred, separated into tubes and stored at $-80{ }^{\circ} \mathrm{C}$ within $4 \mathrm{~h}$.

\section{Genotyping data}

For both the discovery and replication cohorts, DNA was extracted from leukocytes using the TIANamp ${ }^{\circ}$ Blood DNA Kit (DP348, TianGen Biotech Co, Ltd., China) according to the manufacturer's instructions. DNA concentrations were determined using the Qubit quantification system (Thermo Scientific, Wilmington, DE, USA). Extracted DNA was stored at $-80{ }^{\circ} \mathrm{C}$. Genotyping was carried out with Illumina ASA-750K arrays. Quality control and relatedness filters were performed by PLINK1.9 [58]. Individuals with a high or low proportion of heterozygous genotypes (outliers defined as 3 standard deviations) were excluded [59]. Individuals who had different ancestries (the first two principal components \pm 5 standard deviations from the mean) or related individuals (IBD > 0.185) were excluded [59]. Variants were mapped to the 1000 Genomes Phase 3 v 5 by SHAP EIT $[60,61]$, and then we conducted genome-wide genotype imputation with the 1000 Genomes Phase 3 v5 reference panel by Minimac3 [62, 63]. Genetic variants with imputation accuracy RSQR $>0.3$ and MAF $>0.05$ were included in our analysis. We used the Pan-Asian reference panel, consisting of 502 participants, and SNP2HLA v1.0.3 to impute the HLA region [64-66].

\section{Sequencing and processing of $16 \mathrm{~S}$ rRNA gene data}

Microbial DNA was extracted from faecal samples using the QIAamp ${ }^{\circ}$ DNA Stool Mini Kit per the manufacturer's instructions. DNA concentrations were determined using the Qubit quantification system. The V3-V4 region of the $16 \mathrm{~S}$ rRNA gene was amplified from genomic DNA using primers 341F (CCTACGGGNGGCWGCAG) and 805R (GACTACHVGGGTATCTAATCC). At the step of amplicon generation, $2 \mu \mathrm{L}$ sterile water was used as negative controls in the PCR reaction system. At the subsequent step of sequencing, no sequencing negative controls were included, since no contamination of PCR products was observed. The pooled amplicons were sequenced using MiSeq Reagent Kits v2 on the Illumina MiSeq System with $2 \times 250$ bp pair-end sequencing.

Fastq files were demultiplexed by MiSeq Controller Software. Ultra-fast sequence analysis (USEARCH) was performed to trim the sequence for amplification primers, diversity spacers, sequencing adapters and merged paired-end reads [67]. The low-quality reads (Phred quality scores $\leq 30$ ) were removed. Operational taxonomic units (OTUs) were clustered based on 97\% similarity using UPARSE [68]. We removed the OTUs present only in one sample. OTUs were annotated with Greengenes 13_8 (https://greengenes.secondgenome.com/) [69]. After randomly selecting 10000 reads for each sample, Quantitative Insights into Microbial Ecology (QIIME) software version 1.9.0 was used to calculate alpha diversity (Shannon diversity index, Chaol diversity indices and the observed OTU index and phylogenetic diversity) based on the rarefied OTU counts [70].

\section{Statistical analysis \\ Proportion of variance explained by all SNPS}

We used the GREML method in GCTA to estimate the proportion of variance explained by all SNPs [71]. The taxa were divided into two groups based on whether the taxa were present in ninety percent of participants. Our model was adjusted for age and sex. The power of GREML analysis was calculated with the GCTA power calculator [72]. 


\section{Genome-wide association analysis of gut microbiome features}

For each of the GNHS participants and the replication cohort, we clustered participants based on genus-level relative abundance, estimating the JSD distance and PAM clustering algorithm, and then we defined two enterotypes according to the Calinski-Harabasz index $[34,73]$. We calculated the genetic principal components of ancestry from genome-wide genetic variants to estimate the population structure. PLINK 1.9 was used to perform a logistic regression model for enterotypes and taxa present in fewer than ninety percent, adjusted for age, sex, sequencing batch and the first five genetic principal components of ancestry.

For beta diversity, the analysis for the genome-wide host genetic variants with beta diversity was performed using MicrobiomeGWAS [35], adjusted for covariates including the first five genetic principal components of ancestry, age and sex.

Alpha diversity was calculated after randomly sampling 10,000 reads per sample. For the taxa present in no fewer than $90 \%$ of participants and alpha diversity, we used Z-score normalization to transform the distribution and carried out analysis based on a log-normal model. A mixed linear model-based association (MLMA) test in GCTA was used to assess the association, fitting the first five genetic principal components of ancestry, age, sex and sequencing batch as fixed effects and the effects of all the SNPs as random effects [74-76]. For other taxa present in fewer than $90 \%$ of participants, we transformed the absence/presence of the taxon into binary variables and used PLINK1.9 to perform a logistic model, adjusted for the first five genetic principal components of ancestry, age, sex and sequencing batch. For all the gut microbiome features, the significance threshold was defined as $5 \times 10^{-8} / n$ ( $n$ is the effective number of independent taxa in each taxonomic level) in the discovery stage. QUANTO software was used for power calculations (http://biostats.usc.edu/Quanto.html). We estimated genomic inflation factors with LDSC v1.0.1 at the local server [77].

\section{Genetic correlation of gut microbiome and traits}

We used GCTA to perform a bivariate GREML analysis to estimate the genetic correlation between the gut microbiome and traits in GNHS participants [74, 78]. The gut microbiome was divided into two groups according to the previous description. We used continuous variables for taxa present in no fewer than $90 \%$ of participants. For taxa present in fewer than $90 \%$ of participants, we used binary variables according to the absence/presence of taxa. This analysis included traits such as BMI, FBS, HbA1c, SBP, DBP, HDL-C, LDL-C,
TC and TG. The power of bivariate GREML analysis was calculated with the GCTA power calculator [72].

\section{Constructing polygenic scores for taxa and alpha diversity}

We selected lead SNPs using PLINK v1.9 with the '-clump' command to clump SNPs with a $p$ value $<5 \times$ $10^{-5}$ and $r^{2}<0.1$ within $0.1 \mathrm{cM}$. We used beta coefficients as the weight to construct polygenic scores for taxa and alpha diversity. For alpha diversity and taxa present in no fewer than $90 \%$ of participants, we constructed weighted polygenic scores and performed the analysis on a general linear model with a negative binomial distribution to test for association between the polygenic scores and taxa, adjusted for the first five genetic principal components of ancestry, age, sex and sequencing batch. We used weighted polygenic scores and logistic regression to the absence/presence taxa, adjusted for the same covariates as in the above analysis. Taxa with significance $(p<0.05)$ in the replication cohort were included for further analysis.

\section{The effective number of independent taxa}

As some taxa were correlated with each other, we used an eigendecomposition analysis to calculate the effective number of independent taxa for each taxonomic level $[79,80]$. Matrix $M$ is an $m \times n$ matrix, where $m$ is the number of participants and $n$ is the number of total taxa in the corresponding taxonomic level. Matrix $A$ is the variance-covariance matrix of matrix $M . P$ is the matrix of eigenvectors. $\operatorname{diag}\left\{\lambda_{1}, \lambda_{2}, \cdots, \lambda_{n}\right\}$ is the diagonal matrix composed of the ordered eigenvalues, which can be calculated as

$$
\operatorname{diag}\left\{\lambda_{1}, \lambda_{2}, \cdots, \lambda_{n}\right\}=P^{-1} A P
$$

The effective number of independent taxa can be calculated as

$$
\frac{\left(\sum_{i=1}^{n} \lambda_{i}\right)^{2}}{\sum_{i=1}^{n} \lambda_{i}^{2}}
$$

\section{Bidirectional MR analysis}

In the analysis of the potential causal effect of gut microbiome features on diseases, we used independent genetic variants (selected as part of the polygenic score analysis) as the instrumental variables. For each trait, we excluded instrumental variables that showed a significant association with the trait ( $p<0.05 / n$, where $n$ is number of independent genetic variants). In the analysis of the potential causal effect of diseases on gut microbiome features, we selected genetic variants that were replicated in East Asian populations as instrumental variables. As all instrumental variables were from East Asian populations, we chose independent genetic variants $\left(r^{2}<\right.$ 
0.1 ) based on the GNHS cohort. We identified the best proxy $\left(r^{2}>0.9\right)$ based on the GNHS cohort or discarded the variant if no proxy was available. We used the inverse variance weighted (IVW) method to estimate the effect size. To confirm the robustness of the results, we performed three other MR methods, including weighted median, MR-Egger and MR-PRESSO [81-83]. To assess the presence of horizontal pleiotropy, we performed the MR-PRESSO global test and MR-Egger regression. The magnitude of the effect of the gut microbiome on traits was dependent on the units of traits (Supplementary Table S1). The results of the effects of complex human diseases on the absence/presence of specific gut microbes are presented as the risk of the presence (vs. absence) of the microbe per the log odds difference of the disease. The results of the effects of diseases on other gut microbes were presented as changes in the abundance of taxa (1-SD of log transformed) per the log odds difference of the respective disease.

The statistical significance of the effects of the gut microbiome on traits and diseases was defined as $p<$ $0.0008(0.05 / 62)$. In addition, the statistical significance of the effects of diseases on gut microbiome features was defined as $p<0.05 / n$ (where $n$ is the effective number of independent taxa on the corresponding taxonomic level). The results that could not pass Bonferroni adjustment but $p<0.05$ in all four MR methods were considered potential causal relationships. We performed MR analyses with R v3.5.3.

\section{Pathway analysis}

We used OTUs by QIIME and annotated the variation of functional genes with Phylogenetic Investigation of Communities by Reconstruction of Unobserved States (PICRUSt) [39]. The pathways and diseases were annotated using KEGG [84-86]. We used Spearman's rankorder correlation to investigate the association of the predicted pathway or disease abundance with AFassociated taxa and genus-level taxa. In the heatmap, diseases were clustered with the 'hcluster' function in R. To test whether the non-normalized pathway or disease abundance were associated with each other, we used SPIEC-EASI to test the interaction relationship and then used Cytoscape v3.7.2 to visualize the interaction network $[87,88]$.

\section{Construction of the microbiome risk score}

The microbiome risk score was constructed to validate the accuracy of the association between the predicted disease-related gut microbiome features and the corresponding disease. As we have a large sample size for T2DM cases $(n=217$ cases $)$ in our cohort, we constructed a microbiome risk score of T2DM as an example. We used Spearman's rank-order correlation to select taxa with an absolute value of correlation coefficient higher than 0.2. The score for each taxon abundance in the $<5 \%$ quantile in our study was defined as 0 . For those above 5\%, the score for each taxon showing an inverse association with T2DM was defined as -1 ; the score for each taxon showing a positive association with T2DM was defined as 1 . We then summed values from all taxa. We selected a logistic regression model to estimate the association of the MRS with T2DM risk and a linear model to estimate the association of the MRS with the continuous variables, adjusted for age, sex, dietary energy intake, alcohol intake and BMI at the time of sample collection.

\section{Clustering diseases}

The clustering analysis was carried out with 'cluster' and 'factoextra' for plot in R. We performed the PAM algorithm based on the predicted abundance of diseases or the average relative abundance after Z-score normalization [89]. The PAM algorithm searches $k$ medoids among the observations and then finds the nearest medoids to minimize the dissimilarity among clusters [90]. Given a set of objects $x=\left(x_{1}, x_{2}, \ldots, x_{n}\right)$, the dissimilarity between objects $x_{i}$ and $x_{j}$ is denoted by $\mathrm{d}(i$, $j$ ). The assignment of object $i$ to the representative object $j$ is denoted by $z_{i j}$. $z_{i j}$ is a binary variable and is 1 if object $i$ belongs to the cluster of the representative object $j$. The function to minimize the model is given by

$$
\sum_{i=1}^{n} \sum_{j=1}^{n} d(i, j) z_{i j}
$$

To identify the optimal cluster number, we used the 'pamk' function in $\mathrm{R}$ to determine the optimum average silhouette width. For each object $i$, we defined $N_{i}$ as the average dissimilarity between object $i$ and all other objects within its cluster. For the remaining clusters, $\mathrm{b}(i$, $w$ ) represents the average dissimilarity between $i$ and all objects in cluster $w$. The minimum dissimilarity $M_{i}$ can be calculated by

$$
M_{i}=\min \forall w(b(i, w)) .
$$

The silhouette width for object i can be calculated by

$$
s w_{i}=\frac{M_{i}-N_{i}}{\max \left(M_{i}, N_{i}\right)}
$$

Then, we calculated the average silhouette width for each object. The cluster number is determined by the number at which the average silhouette width is maximum. We estimated the Jaccard similarity coefficient to quantify the cluster difference between groups. The Jaccard similarity coefficient is positively associated with 
the similarity of clusters. Given objects $i$ and $j$, as well as groups $\mathrm{A}$ and $\mathrm{B}$, there are four situations, as follows:

1. S1: in both groups $\mathrm{A}$ and $\mathrm{B}$, objects $i$ and $j$ belong to the same cluster

2. S2: in group A, objects $i$ and $j$ belong to the same cluster; in group B, they belong to different clusters

3. S3: in group A, objects $i$ and $j$ belong to different clusters; in group A, they belong to the same cluster

4. S4: in both groups A and B, objects $i$ and $j$ belong to different clusters

The letters $\mathrm{a}, \mathrm{b}, \mathrm{c}$ and $\mathrm{d}$ represent the numbers of $\mathrm{S} 1$, S2, S3 and S4, respectively. The Jaccard similarity coefficient can be calculated by the following formula:

$$
J=\frac{a}{a+b+c}
$$

\section{Supplementary information}

Supplementary information accompanies this paper at https://doi.org/10. 1186/s40168-020-00923-9.

Additional file 1: Table S1. Transformation of traits in BioBank Japan and taxa in GNHS. Table S2. Required effect size (beta) to reach $80 \%$ of power in GNHS cohort. Table S3 Heritability of taxa, enterotype and alpha diversity. Table S4. Significant genetic correlations of gut microbiome and metabolic traits. Table S5. Significant associations of all taxa with SNPs identified in the discovery stage before adjustment $(p<5 \mathrm{e}$ 8). Table S6. Replication of genetic variants associated with taxa. Table S7. Replication of genetic variants associated with beta diversity. Table S8. Replication of genetic variants associated with alpha diversity. Table S9. Lead SNPS used to construct polygenic scores. Table S10. MR analysis of gut microbiota on traits and diseases. Table S11. MR analysis of diseases on gut microbiota features. Table S12. Spearman's correlation of certain taxa and complex diseases. Table S13. Spearman's correlation of gut microbiota on genus level and characteristics.

Additional file 2: Figure S1. Genome-wide analysis results of taxa. Figure S2. Spearman's correlation of the relative abundance of AFassociated taxa with the relative level of diseases predicted by PICRUSt.

\section{Acknowledgements}

We thank the Westlake University Supercomputer Center for providing computing and data analysis services for the present project.

\section{Authors' contributions}

JSZ, YMC and JW initiated and led the study. JY assisted with the data analyses. FZX, YQF and JSZ analysed the data and wrote the manuscript. TYS and CWL collected the data. ZLJ, ZLM, MLS and WLG analysed the data. All authors read and approved the final manuscript.

\section{Funding}

This study was funded by the National Natural Science Foundation of China (81903316, 81773416), the Zhejiang Ten-thousand Talents Program (101396522001) and the 5010 Program for Clinical Research (2007032) of Sun Yat-sen University (Guangzhou, China). Dr Jun Wang was supported by the National Key Research and Development Program of China (grant number 2018YFC2000500), the Strategic Priority Research Program of the Chinese Academy of Sciences (grant number XDB29020000) and the National Natural Science Foundation of China (grant number 31771481 and 91857101).

\section{Availability of data and materials}

The raw data for 165 rRNA gene sequences are available in the CNSA (https://db.cngb.org/cnsa/) of CNGBdb at accession number CNP0000829. Original R scripts are available in GitHub (https://github.com/hsufengzhe/ microbiome/tree/master). Requests for the metadata from this study can be submitted via email to zhengjusheng@westlake.edu.cn. A proposal is also required for approval.

\section{Ethics approval and consent to participate}

This study was approved by the Ethics Committee of the School of Public Health at Sun Yat-sen University and Ethics Committee of Westlake University, and all participants provided written informed consent.

\section{Consent for publication}

Not applicable.

\section{Competing interests}

The authors declare no conflicts of interest.

\section{Author details}

${ }^{1}$ Zhejiang Provincial Laboratory of Life Sciences and Biomedicine, Key Laboratory of Growth Regulation and Translational Research of Zhejiang Province, School of Life Sciences, Westlake University, Hangzhou, China. ${ }^{2}$ Guangdong Provincial Key Laboratory of Food, Nutrition and Health, Department of Epidemiology, School of Public Health, Sun Yat-sen University, Guangzhou, China. ${ }^{3}$ Institute of Basic Medical Sciences, Westlake Institute for Advanced Study, Hangzhou, China. ${ }^{4}$ Institute for Molecular Bioscience, The University of Queensland, QLD, Brisbane, Australia. ${ }^{5}$ Institute for Advanced Research, Wenzhou Medical University, Wenzhou 325027, Zhejiang, China. ${ }^{6}$ CAS Key Laboratory for Pathogenic Microbiology and Immunology, Institute of Microbiology, Chinese Academy of Sciences, Beijing, China. ${ }^{7}$ MRC Epidemiology Unit, University of Cambridge, Cambridge, UK.

Received: 3 July 2020 Accepted: 13 September 2020

Published online: 08 October 2020

\section{References}

1. Awany D, Allali I, Dalvie S, Hemmings S, Mwaikono KS, Thomford NE, et al Host and microbiome genome-wide association studies: current state and challenges. Front Genet. 2019;9:637. https://doi.org/10.3389/fgene.2018. 00637.

2. Bull MJ, Plummer NT. Part 1: The human gut microbiome in health and disease. Integr Med (Encinitas, Calif). 2014;13(6):17-22.

3. Lynch JB, Hsiao EY. Microbiomes as sources of emergent host phenotypes. Science. 2019;365(6460):1405-9. https://doi.org/10.1126/science.aay0240.

4. Allegretti JR, Mullish BH, Kelly C, Fischer M. The evolution of the use of faecal microbiota transplantation and emerging therapeutic indications. Lancet. 2019;394(10196):420-31. https://doi.org/10.1016/S01406736(19)31266-8.

5. Wong $\mathrm{SH}, \mathrm{Yu}$ J. Gut microbiota in colorectal cancer: mechanisms of action and clinical applications. Nat Rev Gastroenterol Hepatol. 2019. https://doi. org/10.1038/s41575-019-0209-8.

6. Davies NM, Holmes MV, Davey SG. Reading Mendelian randomisation studies: a guide, glossary, and checklist for clinicians. BMJ. 2018;362:k601. https://doi.org/10.1136/bmj.k601

7. Turpin W, Espin-Garcia O, Xu W, Silverberg MS, Kevans D, Smith Ml, et al. Association of host genome with intestinal microbial composition in a large healthy cohort. Nat Genet. 2016;48(11):1413-7. https://doi.org/10.1038/ng. 3693.

8. Wang J, Thingholm LB, Skieceviciene J, Rausch P, Kummen M, Hov JR, et al. Genome-wide association analysis identifies variation in vitamin $D$ receptor and other host factors influencing the gut microbiota. Nat Genet. 2016; 48(11):1396-406. https://doi.org/10.1038/ng.3695.

9. Goodrich JK, Waters JL, Poole AC, Sutter JL, Koren O, Blekhman R, et al. Human genetics shape the gut microbiome. Cell. 2014;159(4):789-99. https://doi.org/10.1016/j.cell.2014.09.053.

10. Goodrich JK, Davenport ER, Beaumont M, Jackson MA, Knight R, Ober C, et al. Genetic determinants of the gut microbiome in UK Twins. Cell Host Microbe. 2016;19(5):731-43. https://doi.org/10.1016/j.chom.2016.04.017.

11. Ganesan K, Chung SK, Vanamala J, Xu B. Causal relationship between dietinduced gut microbiota changes and diabetes: a novel strategy to 
transplant Faecalibacterium prausnitzii in preventing diabetes. Int J Mol Sci. 2018;19(12). https://doi.org/10.3390/ijms19123720.

12. He Y, Wu W, Zheng HM, Li P, McDonald D, Sheng HF, et al. Regional variation limits applications of healthy gut microbiome reference ranges and disease models. Nat Med. 2018;24(10):1532-5. https://doi.org/10.1038/ s41591-018-0164-X.

13. Rothschild D, Weissbrod O, Barkan E, Kurilshikov A, Korem T, Zeevi D, et al. Environment dominates over host genetics in shaping human gut microbiota. Nature. 2018;555(7695):210-5. https://doi.org/10.1038/ nature25973.

14. Duvallet C, Gibbons SM, Gurry T, Irizarry RA, Alm EJ. Meta-analysis of gut microbiome studies identifies disease-specific and shared responses. Nat Commun. 2017:8(1):1784. https://doi.org/10.1038/s41467-017-01973-8.

15. Cheng $S$, Han B, Ding M, Wen $Y, M a M$, Zhang $L$, et al. Identifying psychiatric disorder-associated gut microbiota using microbiota-related gene set enrichment analysis. Brief Bioinform. 2019. https://doi.org/10.1093/ bib/bbz034.

16. Jackson MA, Verdi S, Maxan M-E, Shin CM, Zierer J, Bowyer RCE, et al. Gut microbiota associations with common diseases and prescription medications in a population-based cohort. Nat Commun. 2018;9(1):2655 https://doi.org/10.1038/s41467-018-05184-7.

17. Cao Y, Wang C, Guan K, Xu Y, Su Y-X, Chen YM. Association of magnesium in serum and urine with carotid intima-media thickness and serum lipids in middle-aged and elderly Chinese: a community-based cross-sectional study. Eur J Nutr. 2015;55. https://doi.org/10.1007/s00394-015-0839-8.

18. Sun L-L, Li B-L, Xie H-L, Fan F, Yu W-Z, Wu B-H, et al. Associations between the dietary intake of antioxidant nutrients and the risk of hip fracture in elderly Chinese: A case-control study. Bri J Nutr. 2014;112:1-9. https://doi. org/10.1017/S0007114514002773.

19. Low SK, Takahashi A, Ebana Y, Ozaki K, Christophersen IE, Ellinor PT, et al. Identification of six new genetic loci associated with atrial fibrillation in the Japanese population. Nat Genet. 2017:49(6):953-8. https://doi.org/10.1038/ ng.3842.

20. Suzuki K, Akiyama M, Ishigaki K, Kanai M, Hosoe J, Shojima N, et al. Identification of 28 new susceptibility loci for type 2 diabetes in the Japanese population. Nat Genet. 2019;51(3):379-86. https://doi.org/10.1038/ s41588-018-0332-4

21. Akiyama M, Okada $Y$, Kanai M, Takahashi A, Momozawa $Y$, Ikeda M, et al. Genome-wide association study identifies 112 new loci for body mass index in the Japanese population. Nat Genet. 2017;49(10):1458-67. https://doi.org/ 10.1038/ng.3951.

22. Kanai M, Akiyama M, Takahashi A, Matoba N, Momozawa Y, Ikeda M, et al. Genetic analysis of quantitative traits in the Japanese population links cell types to complex human diseases. Nat Genet. 2018;50(3):390-400. https:// doi.org/10.1038/s41588-018-0047-6.

23. Matoba N, Akiyama M, Ishigaki K, Kanai M, Takahashi A, Momozawa Y, et al. GWAS of smoking behaviour in 165,436 Japanese people reveals seven new loci and shared genetic architecture. Nat Hum Behav. 2019;3(5):471-7. https://doi.org/10.1038/s41562-019-0557-y.

24. Okada Y, Wu D, Trynka G, Raj T, Terao C, Ikari K, et al. Genetics of rheumatoid arthritis contributes to biology and drug discovery. Nature. 2014;506(7488):376-81. https://doi.org/10.1038/nature12873.

25. Lu XF, Wang LY, Chen SF, He L, Yang XL, Shi YY, et al. Genome-wide association study in Han Chinese identifies four new susceptibility loci for coronary artery disease. Nat Genet. 2012;44(8):890. https://doi.org/10.1038/ ng.2337.

26. Marzec J, Mao X, Li M, Wang M, Feng N, Gou X, et al. A genetic study and meta-analysis of the genetic predisposition of prostate cancer in a Chinese population. Oncotarget. 2016;7(16):21393-403. https://doi.org/10.18632/ oncotarget.7250

27. Okada Y, Sim X, Go MJ, Wu JY, Gu D, Takeuchi F, et al. Meta-analysis identifies multiple loci associated with kidney function-related traits in east Asian populations. Nat Genet. 2012;44(8):904-9. https://doi.org/10.1038/ng.2352.

28. Zeng C, Matsuda K, Jia WH, Chang J, Kweon SS, Xiang YB, et al. Identification of susceptibility loci and genes for colorectal cancer risk. Gastroenterology. 2016;150(7):1633-45. https://doi.org/10.1053/j.gastro.2016. 02.076.

29. Zhou X, Chen Y, Mok KY, Zhao O, Chen K, Chen Y, et al. Identification of genetic risk factors in the Chinese population implicates a role of immune system in Alzheimer's disease pathogenesis. Proc Natl Acad Sci. 2018;115(8): 1697. https://doi.org/10.1073/pnas.1715554115.
30. Gan W, Walters RG, Holmes MV, Bragg F, Millwood IY, Banasik K, Chen Y, Du $\mathrm{H}$, lona A, Mahajan A, et al. Evaluation of type 2 diabetes genetic risk variants in Chinese adults: findings from 93,000 individuals from the China Kadoorie Biobank. Diabetologia. 2016:59(7):1446-57.

31. Lim MY, You HJ, Yoon HS, Kwon B, Lee JY, Lee S, et al. The effect of heritability and host genetics on the gut microbiota and metabolic syndrome. Gut. 2017; 66(6):1031-8. https://doi.org/10.1136/gutjnl-2015-311326.

32. Davenport ER. Elucidating the role of the host genome in shaping microbiome composition. Gut Microbes. 2016;7(2):178-84. https://doi.org/10. 1080/19490976.2016.1155022.

33. Davenport ER, Cusanovich DA, Michelini K, Barreiro LB, Ober C, Gilad Y. Genome-wide association studies of the human gut microbiota. PLoS One. 2015;10(11):e0140301. https://doi.org/10.1371/journal.pone.0140301.

34. Arumugam M, Raes J, Pelletier E, Le Paslier D, Yamada T, Mende DR, et al. Enterotypes of the human gut microbiome. Nature. 2011;473(7346):174-80. https://doi.org/10.1038/nature09944.

35. Hua X, Song L, Yu G, Goedert JJ, Abnet CC, Landi MT, et al. MicrobiomeGWAS: a tool for identifying host genetic variants associated with microbiome composition. bioRxiv. 2015:031187. https://doi.org/10.1101/031187.

36. Ruhlemann MC, Degenhardt F, Thingholm LB, Wang J, Skieceviciene J, Rausch $\mathrm{P}$, et al. Application of the distance-based $\mathrm{F}$ test in an mGWAS investigating beta diversity of intestinal microbiota identifies variants in SLC9A8 (NHE8) and 3 other loci. Gut Microbes. 2018;9(1):68-75. https://doi. org/10.1080/19490976.2017.1356979.

37. Bonder MJ, Kurilshikov A, Tigchelaar EF, Mujagic Z, Imhann F, Vila AV, et al. The effect of host genetics on the gut microbiome. Nat Genet. 2016;48(11): 1407-12. https://doi.org/10.1038/ng.3663.

38. Tang WHW, Kitai T, Hazen SL. Gut microbiota in cardiovascular health and disease. Circ Res. 2017;120(7):1183-96. https://doi.org/10.1161/CIRCRESAHA. 117.309715.

39. Langille MGl, Zaneveld J, Caporaso JG, McDonald D, Knights D, Reyes JA, et al. Predictive functional profiling of microbial communities using $16 \mathrm{~S}$ rRNA marker gene sequences. Nat Biotechnol. 2013;31:814. https://doi.org/ $10.1038 / \mathrm{nbt} 2676$

40. Canga Y, Emre A, Yuksel GA, Karatas MB, Yelgec NS, Gurkan U, et al. Assessment of atrial conduction times in patients with newly diagnosed Parkinson's disease. Parkinsons Dis. 2018;2018:2916905. https://doi.org/10. 1155/2018/2916905

41. Ihara M, Washida K. Linking atrial fibrillation with Alzheimer's disease: epidemiological, pathological, and mechanistic evidence. J Alzheimers Dis. 2018;62(1):61-72. https://doi.org/10.3233/JAD-170970.

42. Conen D, Wong JA, Sandhu RK, Cook NR, Lee I-M, Buring JE, et al. Risk of malignant cancer among women with new-onset atrial fibrillation. JAMA Cardiol. 2016;1(4):389-96. https://doi.org/10.1001/jamacardio.2016.0280.

43. Rousseeuw PJ. Silhouettes: A graphical aid to the interpretation and validation of cluster analysis. J Comput Appl Math. 1987;20:53-65.

44. Blekhman R, Goodrich JK, Huang K, Sun Q, Bukowski R, Bell JT, et al. Host genetic variation impacts microbiome composition across human body sites. Genome Biol. 2015;16:191. https://doi.org/10.1186/s13059-015-0759-1.

45. Goodrich JK, Davenport ER, Clark AG, Ley RE. The relationship between the human genome and microbiome comes into view. Annu Rev Genet. 2017; 51:413-33. https://doi.org/10.1146/annurev-genet-110711-155532.

46. Kuehbacher T, Rehman A, Lepage $P$, Hellmig S, Fölsch UR, Schreiber S, et al. Intestinal TM7 bacterial phylogenies in active inflammatory bowel disease. J Med Microbiol. 2008;57(12):1569-76.

47. He X, McLean JS, Edlund A, Yooseph S, Hall AP, Liu S-Y, et al. Cultivation of a human-associated TM7 phylotype reveals a reduced genome and epibiotic parasitic lifestyle. Proc Natl Acad Sci U S A. 2015;112(1):244-9. https://doi.org/10.1073/pnas.1419038112.

48. Bor B, Bedree JK, Shi W, McLean JS, He X. Saccharibacteria (TM7) in the human oral microbiome. J Dental Res. 2019;98(5):500-9. https://doi.org/10. $1177 / 0022034519831671$

49. Cirulli ET, Goldstein DB. Uncovering the roles of rare variants in common disease through whole-genome sequencing. Nat Rev Genet. 2010;11(6):41525. https://doi.org/10.1038/nrg2779.

50. Vinter N, Christesen Amanda MS, Fenger-Grøn M, Tjønneland A, Frost L. Atrial fibrillation and risk of cancer: a Danish population-based cohort study. I Am Heart Assoc. 2018. https://doi.org/10.1161/JAHA.118.009543.

51. Zoja C, Corna D, Rottoli D, Zanchi C, Abbate M, Remuzzi G. Imatinib ameliorates renal disease and survival in murine lupus autoimmune disease. Kidney Int. 2006;70(1):97-103. 
52. Boursi B, Mamtani R, Haynes K, Yang Y-X. Parkinson's disease and colorectal cancer risk-A nested case control study. Cancer Epidemiol. 2016;43:9-14. https://doi.org/10.1016/j.canep.2016.05.007.

53. Xie X, Luo X, Xie M. Association between Parkinson's disease and risk of colorectal cancer. Parkinsonism Relat Disord. 2017;35:42-7. https://doi.org/ 10.1016/j.parkreldis.2016.11.011

54. van Rheenen W, Shatunov A, Dekker AM, McLaughlin RL, Diekstra FP, Pulit $\mathrm{SL}$, et al. Genome-wide association analyses identify new risk variants and the genetic architecture of amyotrophic lateral sclerosis. Nat Genet. 2016; 48(9):1043-8. https://doi.org/10.1038/ng.3622.

55. Lambert JC, Ibrahim-Verbaas CA, Harold D, Naj AC, Sims R, Bellenguez C, et al. Meta-analysis of 74,046 individuals identifies 11 new susceptibility loci for Alzheimer's disease. Nat Genet. 2013;45(12):1452-8. https://doi.org/10. 1038/ng.2802.

56. Pankratz N, Beecham GW, DeStefano AL, Dawson TM, Doheny KF, Factor SA, et al. Meta-analysis of Parkinson's disease: identification of a novel locus, RIT2. Ann Neurol. 2012;71(3):370-84. https://doi.org/10.1002/ana.22687.

57. Zhao L, Wang G, Siegel P, He C, Wang H, Zhao W, et al. Quantitative genetic background of the host influences gut microbiomes in chickens. Sci Rep. 2013;3:1163. https://doi.org/10.1038/srep01163.

58. Purcell S, Neale B, Todd-Brown K, Thomas L, Ferreira MAR, Bender D, et al. PLIN $\mathrm{K}$ : a tool set for whole-genome association and population-based linkage analyses. Am J Hum Genet. 2007:81(3):559-75. https://doi.org/10.1086/519795.

59. Anderson CA, Pettersson FH, Clarke GM, Cardon LR, Morris AP, Zondervan KT. Data quality control in genetic case-control association studies. Nat Protoc. 2010;5(9):1564-73. https://doi.org/10.1038/nprot.2010.116.

60. Delaneau O, Marchini J, Zagury J-F. A linear complexity phasing method for thousands of genomes. Nat Methods. 2011;9:179. https://doi.org/10.1038/ nmeth.1785.

61. Delaneau O, Marchini J, The Genomes Project C, McVean GA, Donnelly P, Lunter $G$, et al. Integrating sequence and array data to create an improved 1000 Genomes Project haplotype reference panel. Nat Commun. 2014;5: 3934. https://doi.org/10.1038/ncomms4934.

62. Das S, Forer L, Schönherr S, Sidore C, Locke AE, Kwong A, et al. Nextgeneration genotype imputation service and methods. Nat Genet. 2016;48: 1284. https://doi.org/10.1038/ng.3656.

63. Clarke L, Fairley S, Zheng-Bradley X, Streeter I, Perry E, Lowy E, et al. The international genome sample resource (IGSR): a worldwide collection of genome variation incorporating the 1000 Genomes Project data. Nucleic Acids Res. 2016. https://doi.org/10.1093/nar/gkw829.

64. Okada Y, Kim K, Han B, Pillai NE, Ong RT, Saw WY, et al. Risk for ACPApositive rheumatoid arthritis is driven by shared HLA amino acid polymorphisms in Asian and European populations. Hum Mol Genet. 2014; 23(25):6916-26. https://doi.org/10.1093/hmg/ddu387.

65. Pillai NE, Okada Y, Saw WY, Ong RT, Wang X, Tantoso E, et al. Predicting HLA alleles from high-resolution SNP data in three Southeast Asian populations. Hum Mol Genet. 2014;23(16):4443-51. https://doi.org/10.1093/ hmg/ddu149.

66. Jia X, Han B, Onengut-Gumuscu S, Chen W-M, Concannon PJ, Rich SS, et al. Imputing amino acid polymorphisms in human leukocyte antigens. PLoS One. 2013;8(6):e64683. https://doi.org/10.1371/journal.pone.0064683.

67. Edgar RC. Search and clustering orders of magnitude faster than BLAST. Bioinformatics. 2010;26(19):2460-1. https://doi.org/10.1093/bioinformatics/ btq461.

68. Edgar RC. UPARSE: highly accurate OTU sequences from microbial amplicon reads. Nat Methods. 2013;10(10):996-8. https://doi.org/10.1038/nmeth.2604.

69. Second Genome, Inc: the Greengenes Databases.http://greengenes. secondgenome.com/.

70. Caporaso JG, Kuczynski J, Stombaugh J, Bittinger K, Bushman FD, Costello EK, et al. QIIME allows analysis of high-throughput community sequencing data. Nat Methods. 2010;7(5):335-6. https://doi.org/10.1038/nmeth.f.303.

71. Lee SH, Wray NR, Goddard ME, Visscher PM. Estimating missing heritability for disease from genome-wide association studies. Am J Hum Genet. 2011; 88(3):294-305. https://doi.org/10.1016/j.ajhg.2011.02.002.

72. Visscher PM, Hemani G, Vinkhuyzen AAE, Chen G-B, Lee SH, Wray NR, et al. Statistical power to detect genetic (Co)variance of complex traits using SNP data in unrelated samples. PLoS Genet. 2014;10(4):e1004269. https://doi.org/ 10.1371/journal.pgen.1004269.

73. Caliński T, Harabasz J. A dendrite method for cluster analysis. Communications in Statistics. 1974;3(1):1-27. https://doi.org/10.1080/ 03610927408827101
74. Yang J, Lee SH, Goddard ME, Visscher PM. GCTA: a tool for genome-wide complex trait analysis. Am J Hum Genet. 2011;88(1):76-82. https://doi.org/ 10.1016/j.ajhg.2010.11.011.

75. Yang J, Zaitlen NA, Goddard ME, Visscher PM, Price AL. Advantages and pitfalls in the application of mixed-model association methods. Nat Genet. 2014;46(2):100-6. https://doi.org/10.1038/ng.2876.

76. Yang J, Benyamin B, McEvoy BP, Gordon S, Henders AK, Nyholt DR, et al. Common SNPs explain a large proportion of the heritability for human height. Nat Genet. 2010;42:565. https://doi.org/10.1038/ng.608.

77. Bulik-Sullivan BK, Loh P-R, Finucane HK, Ripke S, Yang J, Patterson N, et al. LD Score regression distinguishes confounding from polygenicity in genome-wide association studies. Nat Genet. 2015;47(3):291-5. https://doi org/10.1038/ng.3211.

78. Lee SH, Yang J, Goddard ME, Visscher PM, Wray NR. Estimation of pleiotropy between complex diseases using single-nucleotide polymorphism-derived genomic relationships and restricted maximum likelihood. Bioinformatics. 2012;28(19):2540-2. https://doi.org/10.1093/bioinformatics/bts474.

79. Wang H, Zhang F, Zeng J, Wu Y, Kemper KE, Xue A, et al. Genotype-byenvironment interactions inferred from genetic effects on phenotypic variability in the UK Biobank. Sci Adv. 2019;5(8):eaaw3538. https://doi.org/10. 1126/sciadv.aaw3538.

80. Bretherton CS, Widmann M, Dymnikov VP, Wallace JM, Bladé I. The effective number of spatial degrees of freedom of a time-varying field. J Clim. 1999; 12(7):1990-2009. https://doi.org/10.1175/1520-0442(1999)012<1990: Tenosd>2.0.Co;2.

81. Bowden J, Davey Smith G, Haycock PC, Burgess S. Consistent estimation in mendelian randomization with some invalid instruments using a weighted median estimator. Genet Epidemiol. 2016;40(4):304-14. https://doi.org/10. 1002/gepi.21965.

82. Burgess S, Thompson SG. Interpreting findings from Mendelian randomization using the MR-Egger method. Eur J Epidemiol. 2017;32(5): 377-89. https://doi.org/10.1007/s10654-017-0255-X.

83. Verbanck $M$, Chen C-Y, Neale B, Do R. Detection of widespread horizontal pleiotropy in causal relationships inferred from Mendelian randomization between complex traits and diseases. Nat Genet. 2018;50(5):693-8. https:// doi.org/10.1038/s41588-018-0099-7.

84. Kanehisa M, Goto S. KEGG: kyoto encyclopedia of genes and genomes. Nucleic Acids Res. 2000;28(1):27-30. https://doi.org/10.1093/nar/28.1.27.

85. Kanehisa M, Sato Y, Furumichi M, Morishima K, Tanabe M. New approach for understanding genome variations in KEGG. Nucleic Acids Res. 2019. https:// doi.org/10.1093/nar/gky962.

86. Kanehisa M. Toward understanding the origin and evolution of cellular organisms. Protein Sci. 2019. https://doi.org/10.1002/pro.3715.

87. Shannon P, Markiel A, Ozier O, Baliga NS, Wang JT, Ramage D, et al. Cytoscape: a software environment for integrated models of biomolecular interaction networks. Genome Res. 2003;13(11):2498-504. https://doi.org/10. 1101/gr.1239303.

88. Kurtz ZD, Müller CL, Miraldi ER, Littman DR, Blaser MJ, Bonneau RA. Sparse and compositionally robust inference of microbial ecological Networks. PLoS Comput Biol. 2015. https://doi.org/10.1371/journal.pcbi.1004226.

89. Reynolds AP, Richards G, de la Iglesia B, Rayward-Smith VJ. Clustering rules: a comparison of partitioning and hierarchical clustering algorithms. J Mathe Model Algorithms. 2006;5(4):475-504. https://doi.org/10.1007/s10852-0059022-1.

90. Kaufman L, Rousseeuw P. Partitioning around medoids (Program PAM): Wiley; 1990. p. 68-125.

\section{Publisher's Note}

Springer Nature remains neutral with regard to jurisdictional claims in published maps and institutional affiliations. 